\title{
Endoscopic Endonasal Transclival Odontoidectomy for Basilar Invagination: Operative Video and Technical Nuances
}

\author{
James K. Liu ${ }^{1,2,3}$ Vincent N. Dodson ${ }^{1}$ Kevin Zhao ${ }^{1,3}$ Jean Anderson Eloy ${ }^{1,2,3}$
}

${ }^{1}$ Department of Neurological Surgery, Rutgers New Jersey Medical School, Neurological Institute of New Jersey, Saint Barnabas Medical Center, Robert Wood Johnson Barnabas Health, Newark, New Jersey, United States

2 Department of Otolaryngology-Head and Neck Surgery, Rutgers New Jersey Medical School, Neurological Institute of New Jersey, Saint Barnabas Medical Center, RWJ Barnabas Health, Newark, New Jersey, United States

${ }^{3}$ Center for Skull Base and Pituitary Surgery, Rutgers New Jersey Medical School, Neurological Institute of New Jersey, Saint Barnabas Medical Center, RWJ Barnabas Health, Newark, New Jersey, United States

\author{
Address for correspondence James K. Liu, MD, Department of \\ Neurological Surgery, Rutgers New Jersey Medical School, \\ Neurological Institute of New Jersey, Saint Barnabas Medical Center, \\ RWJ Barnabas Health, 90 Bergen Street, Suite 8100, Newark, \\ NJ 07103, United States (e-mail: james.liu.md@rutgers.edu).
}

J Neurol Surg B 2021;82(suppl S1):S16-S18.
Abstract
Keywords
- endoscopic endonasal approach
- transclival odontoidectomy
- endoscopic skull base surgery
- basilar invagination
- craniovertebral junction
- operative video

Basilar invagination is a congenital or acquired craniovertebral junction abnormality where the tip of the odontoid process projects through the foramen magnum which can cause severe symptomatic compression of the brainstem and spinal cord. If left untreated, patients can develop progressive quadriparesis. Traditionally, basilar invagination can be treated with cervical traction and posterior stabilization. However, in irreducible cases, anterior decompression via a transoral or endonasal approach may be necessary. In this operative video, we demonstrate an endoscopic endonasal transclival approach for odontoidectomy to successfully treat a 37-year-old female with severe basilar invagination causing symptomatic compression on the cervicomedullary junction resulting in unsteady gait and motor weakness. The patient had KlippelFeil syndrome where the $\mathrm{C} 1$ arch was assimilated to the foramen magnum and transclival drilling was needed to adequately access the odontoid process for removal.

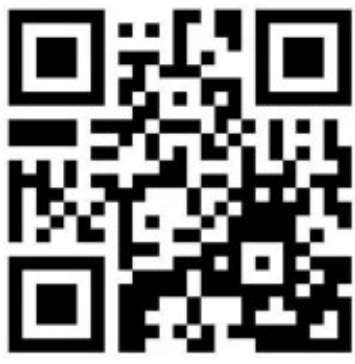

received

June 25,2020

accepted

June 25, 2020

published online

December 2, 2020
Conflict of Interest

None declared.

www.thieme.com/skullbasevideos

www.thieme.com/jnlsbvideos
DOI https://doi.org/ $10.1055 / \mathrm{s}-0040-1715522$ ISSN 2193-6331.

\footnotetext{
(c) 2020. The Author(s).

This is an open access article published by Thieme under the terms of the Creative Commons Attribution-NonDerivative-NonCommercial-License, permitting copying and reproduction so long as the original work is given appropriate credit. Contents may not be used for commercial purposes, or adapted, remixed, transformed or built upon. (https://creativecommons.org/ licenses/by-nc-nd/4.0/) Georg Thieme Verlag KG, Rüdigerstraße 14, 70469 Stuttgart, Germany
} 
A second-stage posterior occipitocervical stabilization and fusion was performed the following day. Immediate postoperative imaging showed excellent decompression of the cervicomedullary junction. Postoperatively, the patient had significant improvement in gait and motor strength in all extremities, and was ambulating independently without assistance at 1 year after surgery. The endoscopic endonasal transclival odontoidectomy is a useful strategy to treat severe irreducible basilar invagination causing symptomatic neural compression. The surgical technique and nuances are described in a step-by-step fashion in this illustrative operative video.

The link to the video can be found at: https://youtu.be/HL4K7KqJEJM.

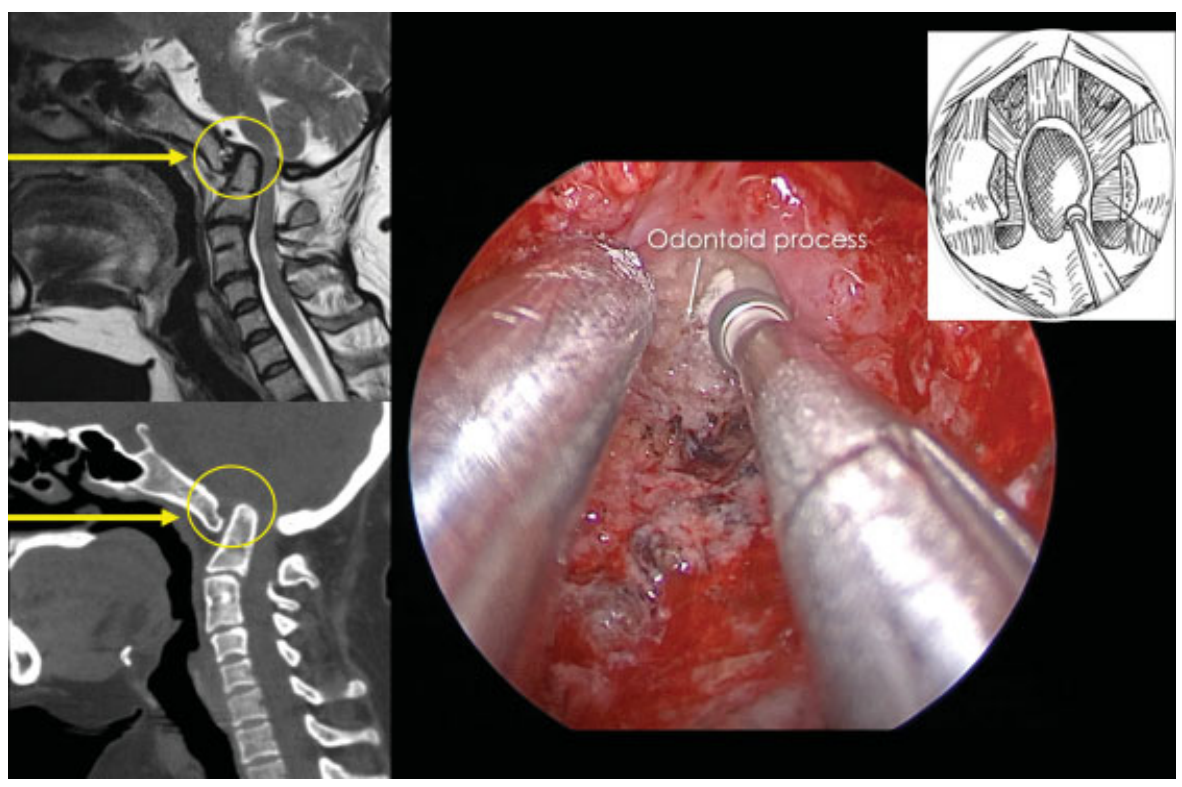

Fig. 1 Top left: Preoperative T2 weighted sagittal MRI demonstrating severe basilar invagination with the odontoid process compressing the cervicomedullary junction and narrowing of the foramen magnum. Bottom left: Preoperative CT sagittal demonstrating the anterior arch of $\mathrm{C} 1$ assimilated to the foramen magnum and the $\mathrm{C} 3$ and $\mathrm{C} 4$ vertebral bodies were fused consistent with Klippel-Feil syndrome. Right: Intraoperative photograph showing endoscopic drilling of the odontoid process using eggshell technique. CT, computed tomography; MRI, magnetic resonance imaging. 

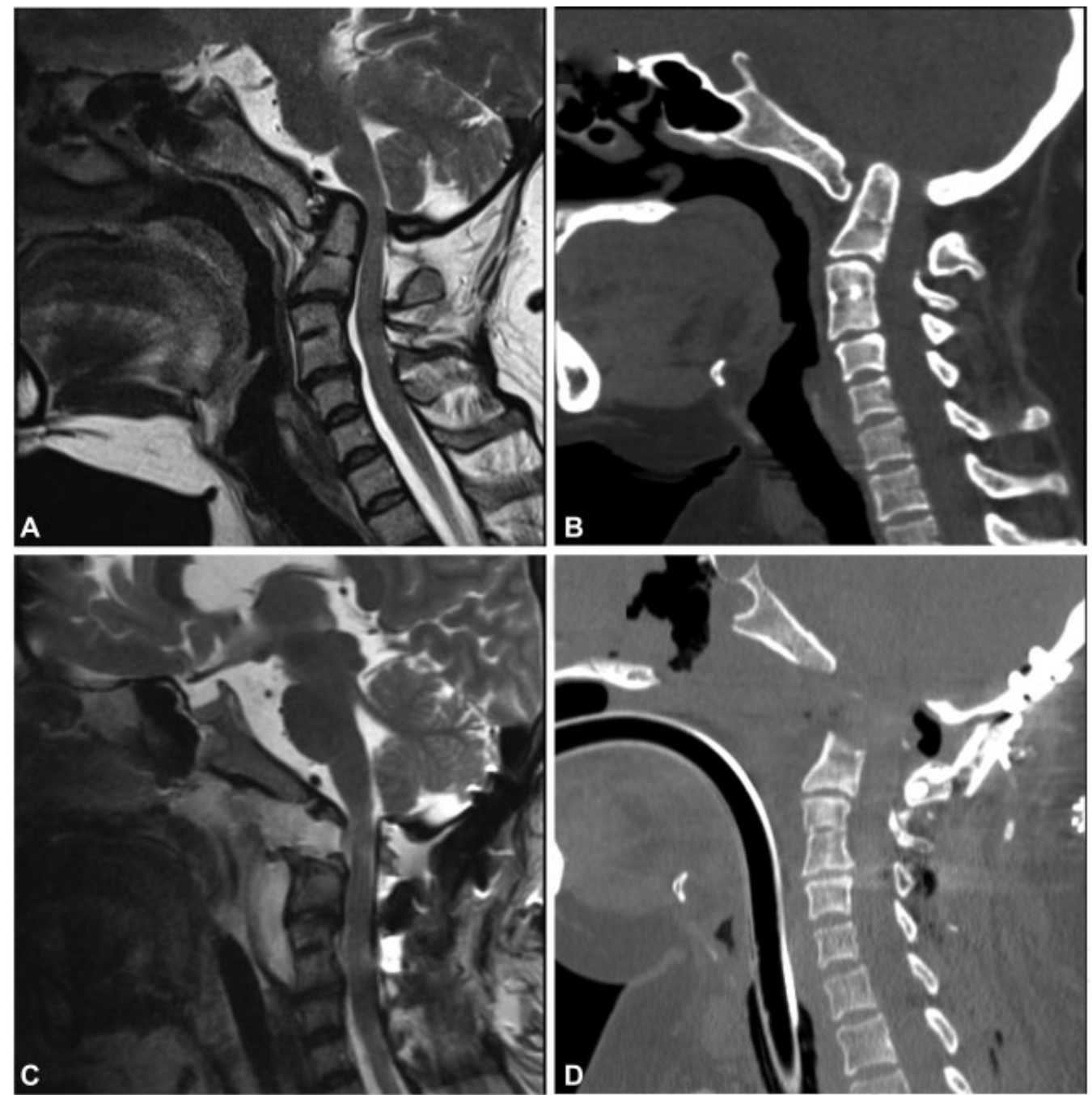

Fig. 2 (A and B) Preoperative T2-weighted sagittal MRI (A) and sagittal CT (B) demonstrating severe basilar invagination with the odontoid process compressing the cervicomedullary junction and narrowing of the foramen magnum. (C and D) Postoperative T2-weighted sagittal MRI (C) and sagittal CT (D) demonstrating excellent decompression of the cervicomedullary junction and occipitocervical instrumented stabilization. $\mathrm{CT}$, computed tomography; MRI, magnetic resonance imaging. 